\title{
A DNS study of the ignition of lean PRF/air mixtures with temperature inhomogeneities under high pressure and intermediate temperature
}

\author{
Seung Ook Kim ${ }^{\mathrm{a}}$, Minh Bau Luong ${ }^{\mathrm{a}}$, Jacqueline H. Chen ${ }^{\mathrm{b}}$, Chun Sang Yoo ${ }^{\mathrm{a}, *}$ \\ ${ }^{a}$ School of Mechanical and Nuclear Engineering, Ulsan National Institute of Science and Technology, Ulsan \\ 689-798, Republic of Korea \\ ${ }^{b}$ Combustion Research Facility, Sandia National Laboratories, Livermore, CA 94551-0969, USA
}

\section{Abstract}

Two-dimensional direct numerical simulations (DNSs) of ignition of lean primary reference fuel $(\mathrm{PRF}) /$ air mixtures at high pressure and intermediate temperature near the negative temperature coefficient (NTC) regime were performed with a 116 species-reduced mechanism to elucidate the effects of fuel composition, thermal stratification, and turbulence on PRF homogeneous charge compression-ignition (HCCI) combustion. In the DNSs, temperature and velocity fluctuations are superimposed on the initial scalar fields with different PRF compositions. In general, it was found that the mean heat release rate increases slowly and the overall combustion occurs rapidly with increasing thermal stratification regardless of the fuel composition. In addition, the effect of the fuel composition on the ignition characteristics of PRF/air mixtures was found to be significantly reduced with increasing thermal stratification. Chemical explosive mode (CEM) and displacement speed analyses verified that nascent ignition kernels induced by hot spots due to a high degree of thermal stratification usually develop into deflagrations rather than spontaneous auto-ignition at reaction fronts and as such, the mean heat release rate becomes more distributed over time. These analyses also revealed that the fuel composition effect vanishes as the degree of thermal stratification is increased because the deflagration mode of combustion, of which propagation characteristics are nearly identical for different PRF/air mixtures, becomes more prevailing with increasing degree of thermal stratification. Ignition Damköhler number was proposed to quantify the successful development of deflagrations from nascent ignition kernels; for cases with large ignition Damköhler number, turbulence with high intensity and short timescale can advance the overall combustion by increasing the overall turbulent flame area instead of homogenizing initial mixture inhomogeneities. Keywords: Homogeneous charge compression ignition (HCCI), Direct numerical simulation (DNS), Primary reference fuel (PRF), Negative temperature coefficient (NTC) regime, Ignition 


\section{Introduction}

The fundamental ignition characteristics of various fuel/air mixtures under lean, dilute, elevated pressure, and relatively low temperature have been widely investigated due to their practical relevance to homogeneous charge compression-ignition (HCCI) engine combustion [1-14]. An HCCI engine and its many variants have been considered as one of the most probable alternatives to the conventional internal combustion engines due to its potential to provide high diesel-like efficiency with very low pollutant emissions [1-14]. However, the development of prototype HCCI engines remains challenging because of their significant drawbacks in preventing an excessive pressure rise rate (PRR) under high-load conditions and controlling precise ignition timing of HCCI combustion. In general, the excessive PRR occurs due to volumetric auto-ignition throughout an HCCI engine cylinder. Moreover, HCCI engines have no explicit ignition method such that ignition timing in HCCI combustion is primarily governed by the chemical kinetics of fuel/air mixture, which depend highly on overall mixture composition, temperature, and pressure. Therefore, there have been many attempts to control the ignition timing and to alleviate the excessive PRR in HCCI combustion by applying different fuel injection strategies, fuel preparation, and thermal management including exhaust gas recirculation (EGR) [1-14].

Several computational studies of HCCI combustion using multi-dimensional direct numerical simulations (DNSs) have been conducted to elucidate the fundamental combustion characteristics of various fuel/air mixtures under HCCI conditions including hydrogen [15-19], dimethyl ether (DME) [20, 21], n-heptane [22], iso-octane [23], primary reference fuel (PRF) [24], ethanol [25], and biodiesel [26]. From these studies, the general characteristics of HCCI combustion have been elucidated; thermal stratification in an HCCI engine cylinder can spread out the PRR under high-load conditions by changing a combustion mode of spontaneous auto-ignition into a mixed combustion mode of spontaneous auto-ignition and deflagration [15-18, 22, 23]. In the presence of large temperature fluctuations, auto-ignition of hotter mixtures first occurs and evolves into deflagration waves, which spread to the unburnt mixtures sequentially until the remaining charge

${ }^{*}$ Corresponding author.

Email address: csyoo@unist.ac.kr (Chun Sang Yoo) 
auto-ignites simultaneously. In general, the speed of the deflagration waves is much less than that of the spontaneous auto-ignition fronts and hence, the overall combustion is temporally spread out, resulting in a reduction of peak PRR.

In many previous DNS studies [15-19], the ignition characteristics of hydrogen/air mixtures exhibiting only one-stage ignition were investigated and as such, the effect of the negative-temperature coefficient (NTC) regime on HCCI combustion was not appreciated. The NTC regime usually appears as a result of the low-temperature oxidation of large hydrocarbon fuels exhibiting two-stage ignition. The effect of the NTC regime on HCCI combustion was first investigated by Yoo et al. [22] using 2-D DNSs of the ignition of a lean $n$-heptane/air mixture with different means and rootmean-squares (RMSs) of temperature. Recently, El-Asrag and Ju [20, 21] investigated the effects of EGR and temperature/mixture stratification on the ignition of synthetic DME by adding $\mathrm{H}_{2} \mathrm{O}_{2}$ and NO to the initial mixtures. Bansal and Im [18], and Bhagatwala et al. [25] also investigated the effect of the equivalence ratio fluctuations on HCCI combustion and found that the equivalence ratio fluctuations enhance HCCI combustion together with temperature fluctuations when they are uncorrelated and retard it when negatively correlated.

It is of interest to note that in a previous DNS study of the ignition of PRF/air mixtures at elevated pressure and high temperature [24], it was found that the effect of different fuel composition on the overall combustion vanishes with increasing temperature inhomogeneities. This is primarily attributed to the predominant deflagration mode of combustion at the reaction fronts and the nearly-identical propagation characteristics of different PRF/air deflagrations. It was also found that turbulence with large intensity and short timescale can effectively homogenize the initial mixtures, rendering the overall combustion to occur by spontaneous auto-ignition.

However, the homogeneous ignition delays of different PRF/air mixtures under high pressure and intermediate temperature conditions, which are more relevant to practical HCCI combustion, show a big disparity among the mixtures (see Fig. 1) and hence, it may be expected that the effect of fuel composition may not vanish even with large temperature fluctuations. Moreover, the early phase of ignition of a fuel/air mixture with very large temperature fluctuation may not be affected by turbulence [23]. In many previous studies [16, 17, 19, 22-24], it was found that turbulence is likely to homogenize the initial mixtures in HCCI combustion and hence, retard its overall combustion. On the contrary, it was also found $[23,25]$ that turbulence may enhance the 
overall spark-assisted compression ignition (SACI) combustion. These DNS results imply that the effect of turbulence on HCCI/SACI combustion may be inconclusive.

Therefore, the objective of the present study is to understand and compare the ignition characteristics of different $\mathrm{PRF} /$ air mixtures at high pressure and intermediate temperature near the NTC regime. For this purpose, we perform 2-D DNSs by varying three key parameters: 1) the fuel composition, 2) the initial temperature fluctuation, and 3) the initial turbulence intensity. Note that both of the initial temperature and composition fluctuations play an important role in HCCI combustion [10, 26]; in general, the equivalence ratio fluctuations enhance HCCI combustion together with temperature fluctuations when they are uncorrelated and retard it when negatively correlated. In the present study, however, we focus only on the effects of temperature fluctuations and turbulence rather than that of equivalence ratio fluctuations because large equivalence ratio fluctuations may be equivalent to large temperature fluctuations if the initial temperature of mixture is near or above the NTC regime. Note that PRF is a fuel mixture of pure $n$-heptane and iso-octane; for instance, PRF 80 is comprised of $80 \%$ iso-octane and $20 \% n$-heptane by liquid volume.

\section{Numerical methods and initial conditions}

For the present DNSs, the Sandia DNS code, S3D [27, 28], is used with a 116-species PRF/air reduced chemistry [24]. S3D solves compressible Navier-Stokes, continuity, total energy, and species continuity equations using a fourth-order, low storage, explicit Runge-Kutta method for time integration [29] and an eighth-order central differencing scheme for spatial discretization [30] with a tenth-order filter. CHEMKIN and TRANSPORT software libraries are linked with S3D to calculate reaction rates and thermodynamic and mixture-averaged transport properties. Details of the numerical algorithm and its implementation are provided in [27]. As in previous DNS studies of HCCI combustion [22-24, 26], periodic boundary conditions are applied in all directions and as such, ignition of $\mathrm{PRF} /$ air mixtures occurs at constant volume.

The skeletal and reduced mechanisms for PRF oxidation were developed for a previous DNS study of ignition of PRF/air mixtures under HCCI condition [24] based on the detailed LLNL mechanism [31, 32], using a strategy combining directed relation graph (DRG)-based methods, isomer lumping, and timescale analysis [24, 33-36]. The skeletal and reduced mechanisms were 
validated under various $\mathrm{PRF}$ composition, pressure, and temperature conditions. Readers are referred to [24] for details of the reduced mechanism.

For all DNSs, we adopted the initial uniform equivalence ratio, $\phi_{0}$, of 0.4 , pressure, $p_{0}$, of 40 atm, and mean temperature, $T_{0}$, of $850 \mathrm{~K}$, respectively. $p_{0}$ of $40 \mathrm{~atm}$ and $T_{0}$ of $850 \mathrm{~K}$ represent high pressure and intermediate temperature near the NTC regime to approximate the conditions in a hydrocarbon-fueled HCCI engine at the top dead center. Under the present initial conditions, the homogeneous ignition delays, $\tau_{i g}^{0}$, of PRF50 and PRF80 are found to be 2.5 and $6.3 \mathrm{~ms}$, respectively. Henceforth, $\tau_{i g}$ represents the time at which the maximum mean heat release rate (HRR) occurs for all simulations. The superscript 0 denotes the 0 -D simulation. A total of ten different DNSs were performed in two dimensions by changing the initial physical conditions: different fuel compositions; temperature fluctuation RMS, $T^{\prime}$; and turbulence velocity fluctuation, $u^{\prime}$.

The initial turbulent flow field is prescribed by an isotropic kinetic energy spectrum function as in $[15-17,37-39]$. The most energetic length scale of turbulence, $l_{e}$, is $1.25 \mathrm{~mm}$. Note that the largest velocity fluctuations in real engines are about $5 \mathrm{~m} / \mathrm{s}$ such that $u^{\prime}$ of 0.5 and $2.5 \mathrm{~m} / \mathrm{s}$ adopted in the present study is quite comparable to that in real engines. Note also that the turbulence timescale in real HCCI engines is $\sim O(1 \mathrm{~ms})$ such that the turbulence timescale in this study, defined as $\tau_{t}=l_{e} / u^{\prime}$, is representative of HCCI combustion. Similar to the initial turbulence field, the initial temperature fluctuation is generated by a temperature spectrum function. For all cases, the most energetic length scale of the temperature fluctuation, $l_{T e}$, of $1.25 \mathrm{~mm}$ is selected such that identical $l_{e}$ and $l_{T e}$ can produce the most effective turbulent mixing as found in [23]. Table 1 shows the details of the physical and numerical parameters. Note that temperature and turbulence fields in the present study are uncorrelated as shown in Fig. 2.

From experiments of HCCI engine [40], it was found that $T^{\prime}$ is approximately $15 \sim 20 \mathrm{~K}$ without thermal management of initial mixture. Therefore, $T^{\prime}=60$ or $120 \mathrm{~K}$ considered in the present study is somewhat large compared to that in real engines. However, these large $T^{\prime}$ can be achieved through direct injection or delayed-fuel injection in HCCI combustion. Furthermore, the spark ignition in SACI combustion [23] and the injection of highly-reactive fuel into an engine cylinder in reactivity controlled compression ignition (RCCI) combustion [41] can significantly reduce the ignition timing as large $T^{\prime}$ in the present study, and hence, it is also important to understand the characteristics of HCCI combustion with large $T^{\prime}$. 
It is of interest to note that 2-D turbulence without vortex stretching and tilting is different from 3-D turbulence. However, it is nearly impossible to do a parametric 3-D DNS study due to its great computational expense and hence, it is still valuable to investigate HCCI combustion using 2-D turbulence with a wide range of spectrum of time- and length-scales by systematically varying important parameters such as temperature and equivalence ratio fluctuations. Moreover, it was found from previous studies [15-18, 22-24] that turbulent mixing plays a secondary role in HCCI combustion compared to temperature stratifications. In addition, it was found from $[19,25]$ that 3-D turbulence may retard/enhance the overall HCCI/SACI combustion slightly more than 2-D turbulence by homogenizing the initial mixtures/increasing turbulent flame area but its effect on overall HCCI/SACI combustion is not significant.

As in previous studies [22-24], a square box of $3.2 \times 3.2 \mathrm{~mm}^{2}$ is chosen for the computational domain, which is discretized with 640 or 1280 grid points, $N$, for the cases in Table 1 . In 2-D DNSs, the turbulent Reynolds numbers based on $u^{\prime}$ and the integral length scale for cases with $u^{\prime}$ $=0.5$ and $2.5 \mathrm{~m} / \mathrm{s}$ are 69 and 347 , respectively. The corresponding Kolmogorov length scales, $\eta_{K}$, are approximately 17.5 and $5.3 \mu \mathrm{m}$, respectively. For all DNS cases, therefore, the Kolmogorov length scale is resolved with at least a grid point [42]. In addition, the thinnest reaction layers in 2-D DNSs are resolved with at least $12 \sim 18$ grid points and as such, turbulence and scalar fields are well resolved in the DNSs. All of the DNSs were performed either on the Cray XC30 at National Energy Research Scientific Computing Center or on the Cray XK7 at Oak Ridge National Laboratory.

\section{Effect of fuel composition and $T^{\prime}$}

In the first parametric study, six different DNS cases (Cases 1-6) are simulated (see Table 1 for detailed parameters of the DNSs) to elucidate the combined effect of the initial fuel composition and temperature fluctuation on the ignition of lean PRF/air mixtures. Different degrees of temperature fluctuation for different PRF/air mixtures are chosen: i.e., $T^{\prime}=15,60$, and $120 \mathrm{~K}$.

\subsection{Overall ignition characteristics}

Figure 3 shows the temporal evolution of mean pressure, $\bar{p}$, mean HRR, $\bar{q}$, and mean PRR, $\bar{p}$, with different levels of temperature fluctuations for different PRFs (Cases 1-6), where "mean" represents the spatial average over the whole simulation domain. Note that the 0-D ignition delay 
of the PRF50/air mixture $\left(\tau_{i g}^{0}=2.5 \mathrm{~ms}\right)$ is chosen as the reference time; $\overline{\dot{q}}$ for the cases with $T^{\prime}$ $=15 \mathrm{~K}$ and $0-\mathrm{D}$ cases are divided by 5 . Several ignition characteristics of the PRF/air mixtures are to be noted. First, $\bar{p}$ increases more slowly and $\bar{q}$ is more distributed over time with increasing $T^{\prime}$ for all fuel compositions, similar to the results in previous studies [15-17, 22-24]. Moreover, the shapes of the overall combustion for Cases 1 and 4 with small $T^{\prime}$ are nearly identical to the corresponding 0-D ignitions. This is because the PRF50/air and PRF80/air mixtures exhibit nearly constant ignition delays at intermediate temperatures around $850 \mathrm{~K}$ as shown in Fig. 1, implying that mixtures with small $T^{\prime}$ are not able to take any advantage of temperature fluctuations to advance the overall combustion $[22,24]$.

Second, overall combustion occurs rapidly and the peak $\bar{q}$ is reduced with increasing $T^{\prime}$ for the same PRF/air mixture, which results in prolonging the vigorous combustion phase. Similar results were also observed from the DNSs of hydrogen, $n$-heptane, $i$ so-octane, and PRF HCCI combustion $[16,17,22-24]$. This is primarily attributed to the occurrence of deflagrations by the large degree of $T^{\prime}$, leading to smoothing of $\bar{q}$ and fast occurrence of overall combustion. As discussed earlier, large $T^{\prime}$ generally induces a mixed mode of deflagration and spontaneous auto-ignition, while small $T^{\prime}$ leads to spontaneous auto-ignition throughout the entire domain.

Third, for cases with the same $T^{\prime}$, the effect of fuel composition is significantly reduced with increasing $T^{\prime}$. For cases with small $T^{\prime}$ (Cases 1 and 4 ), $\tau_{i g}$ increases significantly with increasing iso-octane volume percentage in the PRF as can be expected from their $\tau_{i g}^{0}$; i.e., $\tau_{i g}^{0}=2.5$ and 6.3 ms for PRF50 and 80, respectively. However, for cases with large $T^{\prime}$ (Cases 3 and 6), the difference between $\tau_{i g}$ of the PRF/air mixtures is significantly decreased; i.e., $\tau_{i g} / \tau_{i g}^{0}=0.6$ and 0.8 for PRF50 and 80 , respectively. This result implies that even at intermediate $T_{0}$, the effect of different fuel compositions of PRFs on HCCI combustion may vanish with increasing $T^{\prime}$, similar to the PRF HCCI combustion at high $T_{0}[24]$.

To further identify the overall combustion characteristics of the 2-D DNS cases, the temporal evolutions of the mean mass fractions of important species and the mean HRR are shown in Fig. 4 for four representative cases (Cases 1, 3, 4, and 6) together with their corresponding 0-D ignitions. At the first stage ignition, reaction is initiated by the abstraction of $\mathrm{H}$ from a fuel molecule $(\mathrm{RH})$ by $\mathrm{O}_{2}\left(\mathrm{RH}+\mathrm{O}_{2} \rightarrow \mathrm{R}+\mathrm{HO}_{2}\right)$, forming a cycle with $\mathrm{R}+\mathrm{O}_{2}+\mathrm{M} \rightleftarrows \mathrm{RO}_{2}+\mathrm{M}$ to produce $\mathrm{H}_{2} \mathrm{O}$ and alkylperoxide, $\mathrm{O}=\mathrm{ROOH}[31,32,43,44]$. As such, $\mathrm{HO}_{2}$ increases significantly while 
iso-octane and $n$-heptane decreases rapidly during the first stage ignition of the 0-D ignitions as shown in Figs. 4a and d. This low-temperature reaction cycle comes to an end when temperature exceeds a certain value at which the competing reaction, $\mathrm{R}+\mathrm{O}_{2} \rightarrow$ olefin $+\mathrm{HO}_{2}$, terminates the first stage ignition. Subsequent exothermic reactions start to generate olefin and $\mathrm{H}_{2} \mathrm{O}_{2}$, increasing temperature slowly until the chain branching reaction, $\mathrm{H}_{2} \mathrm{O}_{2}+\mathrm{M} \rightarrow \mathrm{OH}+\mathrm{OH}+\mathrm{M}$, becomes important $[31,32,43,44]$. This reaction sequence proceeds rapidly until the increase of temperature becomes enough to initiate the high-temperature chain branching reactions, controlled by $\mathrm{H}+\mathrm{O}_{2}$ $\rightarrow \mathrm{O}+\mathrm{OH}$. This overall description of the HCCI combustion can be readily found from the 0-D ignitions (see Figs. 4a and d): $\mathrm{H}_{2} \mathrm{O}_{2}$ is first accumulated from the first stage ignition, and then vanishes with a rapid increase of $\mathrm{OH}$ during the second stage ignition, which indicates the importance of $\mathrm{H}_{2} \mathrm{O}_{2}$ in determining the second stage ignition delays.

It is also readily observed from Fig. 4 that the temporal evolutions of the important species in Cases 1 and 4 with small $T^{\prime}$ are nearly identical to those of the corresponding 0-D ignitions, which verifies the predominance of the spontaneous auto-ignition mode of combustion during the whole combustion. Unlike the cases with small $T^{\prime}$, however, the overall combustion of Cases 3 and 6 with large $T^{\prime}$ proceeds gradually without two-stage ignition characteristics; iso-octane and $n$-heptane are consumed more gradually; the production of $\mathrm{CO}$ and $\mathrm{CO}_{2}$ are more distributed over time compared with those of the 0-D ignitions; $\mathrm{CO}_{2}$ and $\mathrm{OH}$ do not have any peaks. These results imply that, rather than the spontaneous auto-ignition like the 0-D ignitions, deflagrations with a slow propagation speed can play a critical role in consuming the fuels and generating the products. Local high-temperature mixtures by large $T^{\prime}$ ignite very rapidly and develop into deflagrations such that the deflagration mode of combustion prevails over the entire domain, thereby leading to the temporal spread of HRR. The detailed analysis of localized physicochemical characteristics of HCCI combustion is an ongoing research topic.

As found in previous studies [15-17, 22-24], the deflagration mode of combustion can be dominant for cases with large $T^{\prime}$, and hence, the propagation characteristics of each PRF/air deflagration wave become more important than the chemical kinetics associated with initiating nascent ignition kernels. Moreover, it is found from 1-D simulations that the laminar flame speeds of PRF/air mixtures with different octane numbers are nearly identical $\left(S_{L} \approx 0.2 \mathrm{~m} / \mathrm{s}\right.$ at $p_{0}=40 \mathrm{~atm}$ and $\left.T_{0}=850 \mathrm{~K}\right)$. Therefore, it is reasonable to expect that overall combustion is not very different for 
different PRF/air mixtures, even though $\tau_{i g}^{0}$ are widely different, particularly if $T^{\prime}$ is large enough to induce a sufficient amount of deflagration.

The ignition characteristics of $\mathrm{PRF} /$ air mixtures can be further identified by investigating the instantaneous isocontours of HRR for Cases 1-3 approximately at each $\tau_{i g}$ as shown in Fig. 5 . The HRR, $\dot{q}$, is normalized by the maximum $\dot{q}$ of the corresponding 0-D ignition of the PRF50/air mixture, $\dot{q}_{m}^{0}=553.7 \mathrm{~J} / \mathrm{mm}^{3}$ s. It is readily observed that for small $T^{\prime}$ (Case 1 ), $\dot{q}$ occurs nearly simultaneously over the entire domain as spontaneous ignition. For large $T^{\prime}$ (Case 3), however, high $\dot{q}$ occurs in thin sheets while low levels of heat release exist in between. This suggests that the mixed mode of spontaneous auto-ignition and deflagration occurs in Case 3, which results in the advancement of the overall combustion, the temporal spread of $\overline{\dot{q}}$, and the reduction of peak $\overline{\dot{q}}$.

\subsection{Chemical explosive mode analysis}

The ignition characteristics of $\mathrm{PRF} /$ air mixtures are further identified using the chemical explosive mode analysis (CEMA). CEMA has been developed for systematically detecting critical flame features such as ignition, extinction, and flame fronts and applied to DNSs of lifted flames in heated coflows [45-48], reacting jets in cross-flows [49, 50], and ignition of hydrocarbon fuel/air mixtures under HCCI conditions [22, 24, 51].

CEMA is briefly summarized here and readers are referred to [46] for more details. In general, the local chemical characteristics in chemically reacting flows can thoroughly be accounted for by the Jacobian of the chemical source term, $\mathbf{J}_{\boldsymbol{\omega}}(\equiv \partial \boldsymbol{\omega} / \partial \mathbf{y})$, in the governing equations, where $\mathbf{y}$ is the solution vector of species concentration and temperature, and $\boldsymbol{\omega}$ is the chemical source term. Chemical explosive mode (CEM) is then defined as an eigenmode of $\mathbf{J}_{\boldsymbol{\omega}}$ where the real part of the eigenvalue, $\lambda_{\mathrm{e}}$, is positive [46]. By definition, CEM represents the reciprocal chemical timescale of a local mixture such that a mixture with CEM is destined to explode if it is isolated from any significant loss in heat or radicals. Therefore, CEM can be considered as an intrinsic chemical feature of ignitable mixtures.

To measure the competition between CEMs and the losses, a Damköhler number, $D a_{c}$, is adopted, which is defined as:

$$
D a_{c}=\lambda_{\mathrm{e}} \cdot \chi^{-1}
$$

where scalar dissipation rate, $\chi\left(=2 D|\nabla c|^{2}\right)$, represents a reciprocal characteristic timescale of the diffusion or loss term $[24,46-48,51]$. Note that $c$ and $D$ are the progress variable and the thermal 
diffusivity of local mixture, respectively. $c$ is defined as $c \equiv Y_{c} / Y_{c}^{\mathrm{Eq}}$, where $Y_{c}=Y_{\mathrm{CO}_{2}}+Y_{\mathrm{CO}}$, and $Y_{c}^{\mathrm{Eq}}$ is the corresponding equilibrium value of $Y_{c}$. A mixture with large positive $D a_{c}$ will likely lead to actual ignition. However, a mixture with large negative $D a_{c}$ represents strongly burning flames. Note that $D a_{c}=1$ indicates ignition or extinction states of steady state combustion.

Figure 6 shows isocontours of $\lambda_{\mathrm{e}}, D a_{c}, \chi$, and temperature for Cases 1 and 3 at each $\tau_{i g}$. To clearly show the reaction fronts, $\lambda_{\mathrm{e}}$ and $D a_{c}$ are shown on a logarithmic scale together with the sign of $\lambda_{\mathrm{e}}$ such that large positive values in Figs. 6a and b represent unburned mixtures while large negative values denote burned mixtures. For both cases, the reaction fronts represented by the thick black lines $\left(D a_{c}=1\right)$ separate the burned and unburned mixtures and correspond to either spontaneous ignition or deflagration. It is readily observed from the figures of $D a_{c}$ that for Case 1, the reaction fronts delineate the auto-igniting (red) region with large positive $D a_{c}$ from the post-ignition (blue) region with large negative $D a_{c}$. It is also of interest to note that $D a_{c} \gg O(1)$ upstream of the reaction fronts indicates that the reaction fronts are spontaneous ignition.

For Case 3, however, there exist three distinct regions: an auto-igniting region (red), a postignition region (blue), and a deflagration region (green). The green region represents reaction fronts upstream of the auto-igniting region which coincide with strips where $\chi$ balances $\lambda_{e}$, a feature of deflagration waves propagating through unburned mixtures prior to the occurrence of auto-ignition. These results also indicate that the reaction fronts of Case 3 are deflagration waves rather than spontaneous ignition fronts.

\section{Effect of turbulence}

In most previous DNS studies of HCCI combustion [16, 17, 22-24], it was found that turbulence with short $\tau_{t}$ and large $u^{\prime}$ is more effective at dissipating initial temperature inhomogeneities than that with long $\tau_{t}$ and small $u^{\prime}$. Therefore, overall combustion is retarded while occurring by spontaneous ignition. However, it was also found from [23] that turbulence with short $\tau_{t}$ and large $u^{\prime}$ can advance overall SACI combustion by increasing turbulent flame area. In this section, therefore, the effect of turbulence on the ignition of PRF/air mixtures is elucidated. Four DNSs (Cases 7-10) were performed with greater $u^{\prime}$ for different PRF/air mixtures (see Table 1 for details of the parameters).

Figure 7 shows the temporal evolution of $\bar{p}$ and $\overline{\dot{q}}$ for the additional cases with $u^{\prime}=2.5 \mathrm{~m} / \mathrm{s}$ 
and the corresponding cases with $u^{\prime}=0.5 \mathrm{~m} / \mathrm{s}$ for PRF50 and PRF80. Several points are to be noted from the figure. For cases with $T^{\prime}$ of $60 \mathrm{~K}$, which is relatively small considering that it induces a small range of ignition delays of the initial mixture, turbulence with short $\tau_{t}$ and large $u^{\prime}$ can effectively homogenize the mixtures. Therefore, both $\tau_{i g}$ and the peak of $\overline{\dot{q}}$ are increased and overall, combustion is more apt to occur by spontaneous ignition for both PRF/air mixtures. For cases with a larger $T^{\prime}$ of $120 \mathrm{~K}$, however, $\tau_{i g}$ is reduced and the peak value of $\bar{q}$ is increased with increasing $u^{\prime}$. This is opposite to the cases with small $T^{\prime}$ and what was found in previous studies $[16,17,22-24]$. This result is more similar to the ignition characteristics of SACI combustion [23].

\subsection{Ignition Damköhler number}

To quantify the effect of turbulence on the early ignition characteristics, we introduce an ignition Damöhler number, $D a_{i g}$, which evaluates the overall competition between ignition and turbulence dissipation during the early phase of HCCI combustion:

$$
D a_{i g}=\tau_{t} / \tau_{i g, 10 \%}
$$

where $\tau_{t}$ is the turbulence timescale of the initial turbulence and $\tau_{i g, 10 \%}$ is the lowest $10 \%$ ignition delay of the initial mixture, which represents the shortest ignition timescale of the initial mixtures. If $D a_{i g} \gg O(1)$, the occurrence of nascent ignition kernels and their evolution to deflagrations are nearly independent of turbulence. On the contrary, if $D a_{i g} \lesssim O(1)$, ignition is significantly affected by turbulence. Note that if $D a_{i g} \ll O(1)$, the homogenization of the initial mixture by turbulence is finished much earlier than the corresponding 0-D ignition delay and, as such, overall combustion becomes similar to homogeneous 0-D ignition.

For Cases 2 and $7\left(T^{\prime}=60 \mathrm{~K}\right), D a_{i g}=1.85$ and $0.37\left(\tau_{i g, 10 \%} \approx 1.35 \mathrm{~ms}\right)$, respectively and hence, the development of nascent ignition kernels is more disturbed by turbulence in Case 7; the initial mixture is more homogenized by large $u^{\prime}$ with short $\tau_{t}$; consequently, the overall combustion of Case 7 is retarded as shown in Fig 7 . For Cases 3 and $8\left(T^{\prime}=120 \mathrm{~K}\right)$, however, $D a_{i g}=14.5$ and 2.9 $\left(\tau_{i g, 10 \%} \approx 0.17 \mathrm{~ms}\right)$, respectively such that the early phase of ignition becomes nearly independent of turbulence. Once deflagrations are developed from the ignition kernels, their evolutions can be highly affected by local turbulence. For these cases, the turbulent flames exist within the corrugated flamelet and thin reaction zone regimes $[52,53]$ such that large $u^{\prime}$ of Case 8 advances the overall combustion by increasing turbulent flame area compared to Case 3. The turbulent flame 
regime will further be discussed in the next section. Figure 8 shows the instantaneous isocontours of HRR for Cases $3\left(u^{\prime}=0.5 \mathrm{~m} / \mathrm{s}\right)$ and Case $8\left(u^{\prime}=2.5 \mathrm{~m} / \mathrm{s}\right)$, which readily identify the increase of turbulent flame area by large $u^{\prime}$ of Case 8 . Note that the above $D a_{i g}$ analysis holds for Cases $7-10$ within the range of $0 \sim 20 \%$ of $\tau_{i g}$ in $D a_{i g}$.

It is of interest to note that even for the SACI cases of which mean temperature is above the NTC regime (see Cases 4 and 10 in [23]), the ignition Damköhler number analysis can directly be applied. From the results of the two SACI cases, it is readily observed that the corresponding $D a_{i g}$ of the two cases are $\sim O(10)$. As such, large $u^{\prime}$ of Case 10 results in the advancement of the overall combustion compare to that of Case 4. Note that the high temperature source can be regarded as large $T^{\prime}$ in the present study.

The occurrence of the early deflagration can be identified by the density-weighted displacement speed, $S_{d}^{*}$, which also shows the propagation characteristics of the combustion waves. $S_{d}^{*}$ is defined by $[45,54,55]$ :

$$
S_{d}^{*}=\frac{1}{\rho_{u}\left|\nabla Y_{k}\right|}\left(\dot{\omega}_{k}-\frac{\partial}{\partial x_{j}}\left(\rho Y_{k} V_{j, k}\right)\right)
$$

where $Y_{k}, V_{j, k}$, and $\dot{\omega}_{k}$ denote the species mass fraction, the species diffusion velocity in the $j$ direction, and the net production rate of species $k$, respectively. $\rho_{u}$ is the density of the unburnt mixture calculated from the local enthalpy and fresh mixture conditions [16, 17, 22-24]. The isocontour of $Y_{c}=0.065$ is chosen to evaluate the displacement speed for all cases as in previous studies [22-24]. This particular isocontour coincides approximately with the location of maximum $\dot{q}$ of $\mathrm{PRF} /$ air mixtures.

Figure 9 shows the temporal evolutions of the mean front speed, $\overline{S_{d}^{*}}$, for the DNS cases, all of which are normalized by the corresponding laminar flame speed, $S_{L}$. As in previous studies $[22,23], S_{L}$ was estimated from a transient $1-\mathrm{D}$ reactive simulation. The initial species composition, pressure, and temperature for the 1-D simulations were specified the same as those of the mean values in 2-D DNSs without any fluctuations. A high-temperature ignition source was then added on the initial constant temperature field to generate a combustion wave, which emanates from the source, propagating into the reactive mixture ahead of it. Auto-ignition in the reactive mixture does not occur prior to $\tau_{i g}^{0}$ such that the measured displacement speed, $S_{d}^{*}$, of the combustion wave can be considered as the laminar flame speed, $S_{L}$, at the corresponding pressure. $S_{L}$ is found to be approximately $0.2 \mathrm{~m} / \mathrm{s}$ for all PRF/air mixtures and remain nearly identical during the combustion 
wave propagation.

As found in previous studies [16, 17, 22-24], the mean front speeds show a characteristic ' $U$ ' shape of HCCI combustion. The initial thermal run-away in the nascent ignition kernel results in large $\overline{S_{d}^{*}}$ during the early phase of combustion. In the same way, the simultaneous auto-ignition of the unburned end-gas leads to the sudden increase of $\overline{S_{d}^{*}}$ during the final phase of combustion $[22-24,55]$.

It is also observed that after the initial thermal run-away, $\overline{S_{d}^{*}}$ similar to $S_{L}$ develops earlier with increasing $T^{\prime}$ and the duration of the region with a constant front speed at the bottom of the ' $U$ ' shape also increases with increasing $T$ '. Note that the start of a relatively-constant front speed implies the occurrence of deflagrations. For the cases with large $T^{\prime}$, the start of deflagration mode of combustion coincides regardless of fuel composition and $u^{\prime}$, verifying that for cases with $D a_{i g} \gg O(1)$, the role of turbulent mixing on the development of nascent ignition kernels and their evolution into deflagrations is negligible.

\section{Discussion}

After the successful development of deflagrations from ignition kernels by large $D a_{i g}$, the temporal evolutions of deflagrations depend highly on their propagation characteristics and local turbulence conditions [52]. It is found from the present DNSs that the laminar flame thickness, $l_{L}$, and speed, $S_{L}$, are approximately $0.04 \mathrm{~mm}$ and $0.2 \mathrm{~m} / \mathrm{s}$, respectively. Therefore, for Cases 3 and 6 , $l_{e} / l_{L} \sim O(10)$ and $u^{\prime} / S_{L} \sim O(1)$. For Cases 8 and 10, however, $l_{e} / l_{L} \sim O(10)$ and $u^{\prime} / S_{L} \sim O(10)$. As such, all turbulent flames with large $T^{\prime}$ exist within the corrugated flamelet and thin reaction zone regimes in the regime diagram for premixed turbulent combustion $[52,53]$ as shown in Fig. 10. This implies that large $u^{\prime}$ induces more corrugated flame structures and thus, enhances the turbulent burning rate by increasing turbulent flame area as shown in Fig. 8. Note that for the cases considered in Fig. 10, the occurrence of the ignition kernels and subsequent development of the deflagrations are finished much earlier than one eddy turnover time of turbulence, and hence, $u^{\prime}$ and $l_{e}$ are found to be nearly identical to those of the initial conditions such that the locations of the cases on the turbulent combustion regime remain the same.

In summary, two key parameters should be considered to understand the effect of turbulence on the characteristics of hydrocarbon-fueled HCCI combustion: $D a_{i g}$ and $u^{\prime} / S_{L}$. If $D a_{i g}$ is large 
enough to successfully develop deflagrations in HCCI combustion, overall combustion can be advanced with large $u^{\prime} / S_{L}$ since the corresponding turbulent flames exist within the corrugated flamelet and thin reaction zone regimes. On the contrary, if $D a_{i g}$ is small enough to disturb the early development of ignition kernels, the overall combustion is retarded because turbulence with short $\tau_{t}$ and large $u^{\prime}$ can effectively homogenize the initial mixture fluctuations. It is of great importance to note that 2-D turbulence is qualitatively different from $3-\mathrm{D}$ turbulence such that the findings from the present 2-D DNSs need to be verified and generalized by a parametric 3-D DNS study, which is a future research topic.

The analysis of $D a_{i g}$ together with turbulent flame regime can be applied to SACI combustion; turbulence with large $u^{\prime}$ can advance the overall SACI combustion exhibiting very large $D a_{i g}$ $(\gg 1)$ by spark ignition as in [23]. In the same way, it may be conjectured that 3 -D turbulence in SACI combustion may also advance the overall combustion more than 2-D turbulence, which was observed in [25], because the effect of vortex stretching in 3-D turbulence can locally strengthen $u^{\prime}$ more than 2-D turbulence, resulting in the increase of turbulent flame area. Due to the same vortex stretching effect, 3 -D turbulence with small $D a_{i g}$ may retard the overall HCCI combustion more than 2-D turbulence by homogenizing the initial mixture inhomogeneities [19].

It is worth mentioning that in addition to the DNS studies of HCCI combustion, there have been many attempts to develop and validate turbulent combustion models for large eddy simulations (LES) and Reynolds averaged Navier-Stokes simulations (RANS) based on DNS data. Several models including flamelet-based models [56], probability density function (PDF) based models [57], and conditional moment closure (CMC) based model [58] for HCCI combustion have been proposed. These combustion models show good agreements with DNS results especially for small temperature and composition stratifications. As such, the present DNS database can be further utilized to develop turbulent combustion models for HCCI combustion with large thermal stratifications.

\section{Conclusions}

The effects of PRF composition, $T^{\prime}$, and $u^{\prime}$ on the ignition of lean PRF/air mixtures at high pressure and intermediate temperature are investigated by 2-D DNSs with a 116-species reduced mechanism. The chemical explosive mode and displacement speed analyses verify that, in general, larger $T^{\prime}$ induces greater temporal spreading of the mean HRR regardless of PRF composition 
because the deflagration mode is predominant at the reaction fronts for large $T^{\prime}$. However, spontaneous ignition prevails for small $T^{\prime}$ and, hence, simultaneous auto-ignition occurs throughout the whole domain, resulting in an excessive HRR. The effect of fuel composition on the ignition of $\mathrm{PRF}$ /air mixtures is found to be significantly reduced with increasing $T^{\prime}$ because the deflagration mode prevails at the reaction fronts and the propagation characteristics of these deflagrations are nearly identical.

The ignition Damköhler number, $D a_{i g}$, defined by the ratio of turbulence timescale to the shortest ignition delay of initial mixtures, was proposed to evaluate the effects of turbulence on the early evolution of deflagrations from ignition kernels. For cases with $D a_{i g} \lesssim O(1)$, turbulence with large $u^{\prime}$ can effectively homogenize the mixtures and disturb the evolution of deflagrations from ignition kernels due to the short turbulence timescale compared to the ignition timescale. Therefore, the overall combustion is retarded and more apt to occur by spontaneous ignition. For cases with $D a_{i g} \gg O(1)$, however, the occurrence of nascent ignition kernels and their evolution to deflagrations are not affected by turbulence and the subsequent development of deflagrations occur only within the corrugated flamelet and thin reaction zone regimes of turbulent combustion. As such, turbulence with large $u^{\prime}$ can advance the overall combustion by increasing turbulent flame area.

\section{Acknowledgements}

The work at Ulsan National Institute of Science and Technology was supported by the Basic Science Research Program through the National Research Foundation of Korea funded by the Ministry of Education, Science and Technology (No. 2011-0008201). SOK and MBL were also supported by BK21PLUS funded by the Korea Ministry of Education. The work at Sandia National Laboratories was supported by the Division of Chemical Sciences, Geosciences, and Biosciences, Office of Basic Energy Sciences, and Office of Advanced Scientific Computing Research of the US Department of Energy. JHC was also supported as part of the Combustion Energy Frontier Research Center, an Energy Frontier Research Center funded by the U.S. Department of Energy, Office of Science, Office of Basic Energy Sciences under Award Number DE-SC0001198. Sandia is a multiprogram laboratory operated by Sandia Corporation, a Lockheed Martin Company, for the United States Department of Energy under contract DEAC04-94AL85000. This research used 
resources of the Oak Ridge Leadership Computing Facility at the Oak Ridge National Laboratory, which is supported by the Office of Science of the U.S. Department of Energy under Contract No. DE-AC05-00OR22725 and the National Energy Research Scientific Computing Center, which is supported by the Office of Science of the U.S. Department of Energy under Contract No. DEAC02-05CH11231.

\section{References}

[1] J. E. Dec, Proc. Combust. Inst. 32 (2009) 2727-2742.

[2] M. Yao, Z. Zheng, H. Liu, Prog. Energy Combust. Sci. 35 (2009) 398-437.

[3] R. Reitz, Combust. Flame 160 (2013) 1-8.

[4] X. Lü, D. Han, Z. Huang, Prog. Energy Combust. Sci. 37 (2011) 741-783.

[5] S. Saxena, I. D. Bedoya, Prog. Energy Combust. Sci. 39 (2013) 457-488.

[6] A. Hultqvist, M. Christenson, B. Johansson, M. Richter, J. Nygren, J. Hult, M. Alden, SAE paper (2002) 2002-01-0424.

[7] J. A. Eng, SAE paper (2002) 2002-01-2859.

[8] A. Babajimopoulos, G. A. Lavoie, D. N. Assanis, SAE paper (2003) 2003-01-3220.

[9] M. Sjöberg, J. E.Dec, SAE Trans. paper 115 (2006) 318-334.

[10] J. E. Dec, W. Hwang, M. Sjöberg, SAE Trans. (2006) 2006-01-1518.

[11] J. E. Dec, W. Hwang, SAE Trans. paper (2009) 2009-01-0650.

[12] Y. Yang, J. E. Dec, N. Dronniou, M. Sjöberg, Proc. Combust. Inst. 33 (2011) 3047-3055.

[13] Y. Yang, J. Dec, N. Dronniou, M. Sjberg, W. Cannella, SAE paper (2011) 2011-01-1359.

[14] A. Krisman, E. R. Hawkes, S. Kook, M. Sjöberg, J. E. Dec, Fuel 99 (2012) 45-54.

[15] R. Sankaran, H. G. Im, E. R. Hawkes, J. H. Chen, Proc. Combust. Inst. 30 (2005) 875-882.

[16] J. H. Chen, E. R. Hawkes, R. Sankaran, S. D. Mason, H. G. Im, Combust. Flame 145 (2006) 128-144.

[17] E. R. Hawkes, R. Sankaran, P. Pébay, J. H. Chen, Combust. Flame 145 (2006) 145-159.

[18] G. Bansal, H. G. Im, Combust. Flame 158 (2011) 2105-2112.

[19] R. Yu, X.-S. Bai, Combust. Flame 160 (2013) 1706-1716.

[20] H. A. El-Asrag, Y. Ju, Combust. Theory Modelling 17 (2013) 316-334.

[21] H. A. El-Asrag, Y. Ju, Combust. Flame 161 (2014) 256-269.

[22] C. S. Yoo, T. Lu, J. H. Chen, C. K. Law, Combust. Flame 158 (2011) 1727-1741.

[23] C. S. Yoo, Z. Luo, T. Lu, H. Kim, J. H. Chen, Proc. Combust. Inst. 34 (2013) 2985-2993.

[24] M. B. Luong, Z. Luo, T. Lu, S. H. Chung, C. S. Yoo, Combust. Flame 160 (2013) 2038-2047.

[25] A. Bhagatwala, T. Lu, J. H. Chen, Combust. Flame 161 (2014) 1826-1841.

[26] M. B. Luong, T. Lu, S. H. Chung, C. S. Yoo, Combust. Flame (2014) in press, http://dx.doi.org/10.1016/j.combustflame.2014.05.004. 
[27] J. H. Chen, A. Choudhary, B. D. Supinski, M. DeVries, E. R. Hawkes, S. Klasky, W. K. Liao, K. L. Ma, J. Mellor-Crummey, N. Podhorszki, R. Sankaran, S. Shende, C. S. Yoo, Comput. Sci. Discov. 2 (2009) 015001.

[28] J. H. Chen, Proc. Combust. Inst. 33 (2011) 99-123.

[29] C. A. Kennedy, M. H. Carpenter, R. M. Lewis, Appl. Num. Math. 35 (2000) 117-219.

[30] C. A. Kennedy, M. H. Carpenter, Appl. Num. Math. 14 (1994) 397-433.

[31] H. J. Curran, P. Gaffuri, W. J. Pitz, C. K. Westbrook, Combust. Flame 129 (2002) 253-280.

[32] H. J. Curran, P. Gaffuri, W. J. Pitz, C. K. Westbrook, Combust. Flame 114 (1998) 149-177.

[33] T. Lu, C. K. Law, Proc. Combust. Inst. 31 (2005) 1333-1341.

[34] T. Lu, C. K. Law, Combust. Flame 148 (2007) 117-126.

[35] T. Lu, C. K. Law, Combust. Flame 154 (2008) 153-163.

[36] T. Lu, C. K. Law, C. S. Yoo, J. H. Chen, Combust. Flame 156 (2009) 1542-1551.

[37] C. S. Yoo, Y. Wang, A. Trouvé, H. G. Im, Combust. Theory Modelling 9 (2005) 617-646.

[38] C. S. Yoo, H. G. Im, Combust. Theory Modelling 11 (2007) 259-286.

[39] C. S. Yoo, H. G. Im, Proc. Combust. Inst. 31 (2007) 701-708.

[40] W. Hwang, J. E. Dec, M. Sjöberg, SAE Trans. paper 116 (2007) 2007-01-4130.

[41] S. L. Kokjohn, R. M. Hanson, D. A. Splitter, R. D. Reitz, Int. J. Engine Res. (2011) 209-226.

[42] S. B. Pope, Turbulent flows, Cambridge University Press, 2000.

[43] S. Tanaka, F. Ayala, J. C. Keck, J. B. Heywood, Combust. Flame 132 (2003) 219-239.

[44] C. K. Law, Combustion physics, Cambridge University Press, 2006.

[45] C. S. Yoo, R. Sankaran, J. H. Chen, J. Fluid Mech. 640 (2009) 453-481.

[46] T. Lu, C. S. Yoo, J. H. Chen, C. K. Law, J. Fluid Mech. 652 (2010) 45-64.

[47] C. S. Yoo, E. S. Richardson, R. Sankaran, J. H. Chen, Proc. Combust. Inst. 33 (2011) 1619-1627.

[48] Z. Luo, C. S. Yoo, E. S. Richardson, J. H. Chen, C. K. Law, T. Lu, Combust. Flame 159 (2012) $265-274$.

[49] R. W. Grout, A. Gruber, C. S. Yoo, J. H. Chen, Proc. Combust. Inst. 33 (2011) 1629-1637.

[50] H. Kolla, R. W. Grout, A. Gruber, J. H. Chen, Combust. Flame 159 (2012) 2755-2766.

[51] R. Shan, C. S. Yoo, J. H. Chen, T. Lu, Combust. Flame 159 (2012) 3119-3127.

[52] N. Peters, Turbulent combustion, Cambridge University Press, 2000.

[53] R. Borghi, Prog. Energy Combust. Sci. 14 (1988) 245-292.

[54] C. S. Yoo, H. G. Im, Proc. Combust. Inst. 20 (2005) 349-356.

[55] C. S. Yoo, J. H. Chen, J. H. Frank, Combust. Flame 156 (2009) 140-151.

[56] D. J. Cook, H. Pitch, J. H. Chen, E. R. Hawkes, Proc. Combust. Inst. 31 (2007) 2903-2911.

[57] F. Bisetti, J.-Y. Chen, J. H. Chen, E. R. Hawkes, Combust. Flame 155 (2008) 571-584.

[58] F. Salehi, M. Talei, E. R. Hawkes, C. S. Yoo, T. Lucchini, G. D’Errico, S. Kook, Proc. Combust. Inst. (2014) in press, http://dx.doi.org/10.1016/j.proci.2014.05.035. 


\section{A DNS study of ignition of lean PRF/air mixtures with temperature inhomogeneities under high pressure and intermediate temperature}

\section{List of Tables}

1 Physical and numerical parameters of the DNSs. . . . . . . . . .

\section{List of Figures}

1 0-D ignition delays as a function of initial temperature for different PRF/air mixtures at $p_{0}=40 \mathrm{~atm}$ and of $\phi_{0}=0.4 \ldots \ldots \ldots \ldots \ldots \ldots$

2 Initial temperature (top) and vorticity (bottom) fields of Case $2 \ldots \ldots$

3 Temporal evolutions of the mean pressure and mean HRR (left) and the PRR (right) for Cases 1-3 (PRF50; a \& c) and Cases 4-6 (PRF80; b \& d) . . . . . . . . . . . 20

4 Temporal evolutions of the mean mass fractions of important species and mean HRR for (a) 0-D case of PRF50/air mixture, (b) Case 1, (c) Case 3, (d) 0-D case of PRF $80 /$ air mixture, (e) Case 4 , and (f) Case $6 \ldots \ldots \ldots \ldots$

5 Isocontours of the normalized heat release rate for Cases 1-3 (from left to right) at $t / \tau_{i g}^{0}=1.0,0.94$, and 0.61 , respectively. . . . . . . . . . . 21

6 Isocontours of (a) the timescale of the chemical explosive mode, (b) $D a_{c}$, (c) temperature, and $(\mathrm{d}) \chi$ for Case 1 (top) at $t / \tau_{i g}^{0}=1.0$ and Case 3 (bottom) at $t / \tau_{i g}^{0}=$ 0.61. The solid line denotes the reaction front $\left(D a_{c}=1\right) \ldots \ldots \ldots$

7 Temporal evolutions of the mean pressure and mean HRR for (a) PRF50 and (b)

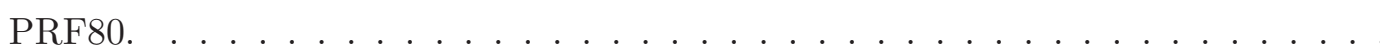

8 Isocontours of the normalized heat release rate for Cases 3 (top) and 8 (bottom). From left to right, $t / \tau_{i g}^{0}=0.36$ and 0.52 , respectively. . . . . . . . . 23

9 Temporal evolution of the mean front speed, $\overline{S_{d}^{*}}$, for (a) Cases 2, 3, 7, and 8 (PRF50) and (b) Cases 5, 6, 9, and $10(\mathrm{PRF} 80) \ldots \ldots \ldots \ldots$

10 The locations of the cases with small $u^{\prime}$ (Cases 3 and 6) and large $u^{\prime}$ (Cases 8 and 10) in the regime diagram for turbulent premixed combustion. . . . . . . . . . 
Table 1: Physical and numerical parameters of the DNSs.

\begin{tabular}{rlcccccc}
\hline Case & Fuel & $T^{\prime}$ & $l_{e}$ & $u^{\prime}$ & $\tau_{t}$ & $\tau_{i g}^{0}$ & $N$ \\
& & $(\mathrm{~K})$ & $(\mathrm{mm})$ & $(\mathrm{m} / \mathrm{s})$ & $(\mathrm{ms})$ & $(\mathrm{ms})$ & \\
\hline 1 & PRF50 & 15 & 1.25 & 0.5 & 2.5 & 2.5 & 640 \\
2 & PRF50 & 60 & 1.25 & 0.5 & 2.5 & 2.5 & 640 \\
3 & PRF50 & 120 & 1.25 & 0.5 & 2.5 & 2.5 & 1280 \\
4 & PRF80 & 15 & 1.25 & 0.5 & 2.5 & 6.3 & 640 \\
5 & PRF80 & 60 & 1.25 & 0.5 & 2.5 & 6.3 & 640 \\
6 & PRF80 & 120 & 1.25 & 0.5 & 2.5 & 6.3 & 1280 \\
7 & PRF50 & 60 & 1.25 & 2.5 & 0.5 & 2.5 & 640 \\
8 & PRF50 & 120 & 1.25 & 2.5 & 0.5 & 2.5 & 1280 \\
9 & PRF80 & 60 & 1.25 & 2.5 & 0.5 & 6.3 & 640 \\
10 & PRF80 & 120 & 1.25 & 2.5 & 0.5 & 6.3 & 1280 \\
\hline
\end{tabular}

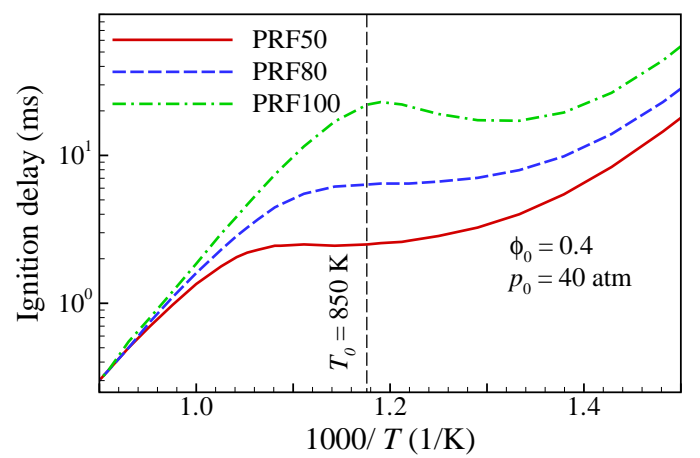

Figure 1: 0-D ignition delays as a function of initial temperature for different PRF/air mixtures at $p_{0}=40$ atm and of $\phi_{0}=0.4$. 

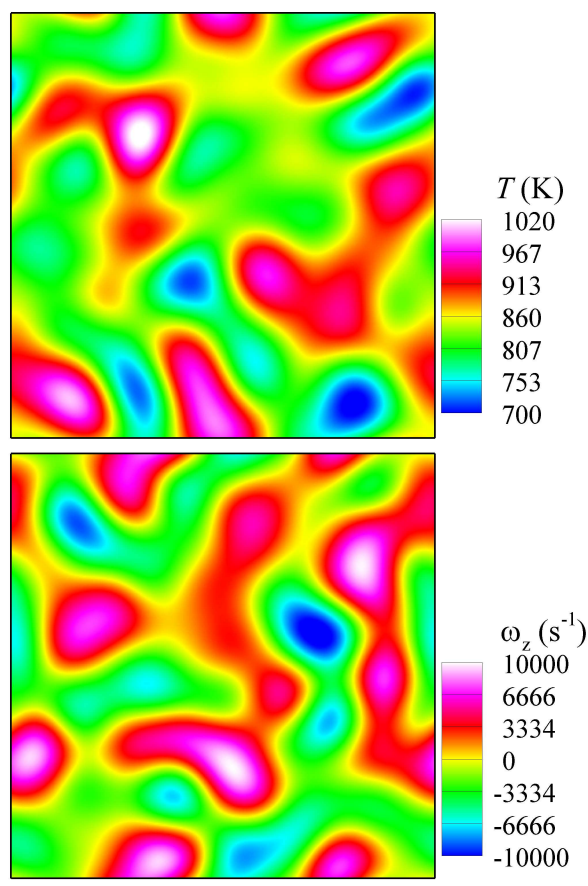

Figure 2: Initial temperature (top) and vorticity (bottom) fields of Case 2.
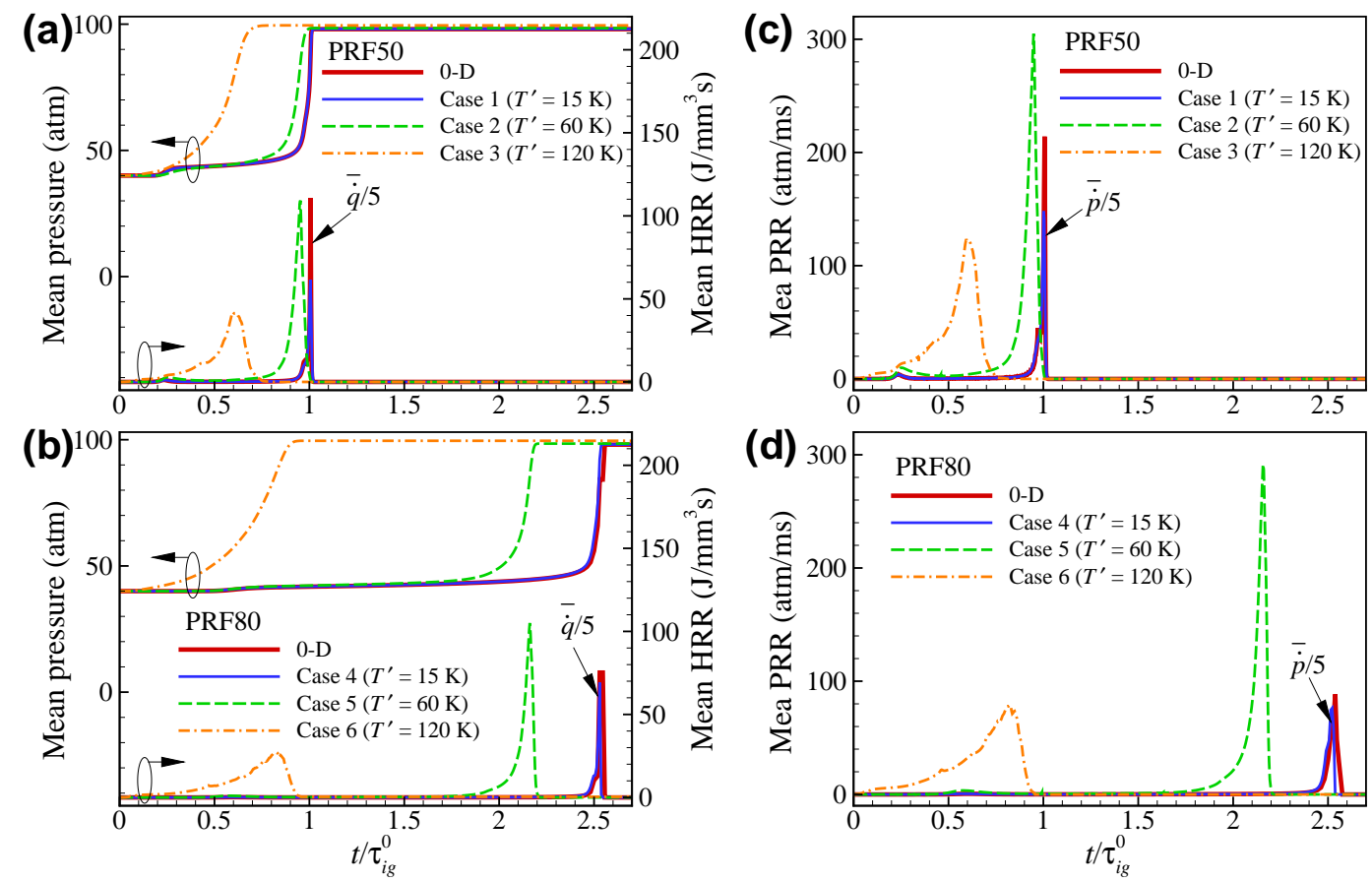

Figure 3: Temporal evolutions of the mean pressure and mean HRR (left) and the PRR (right) for Cases 1-3 (PRF50; a \& c) and Cases 4-6 (PRF80; b \& d). 

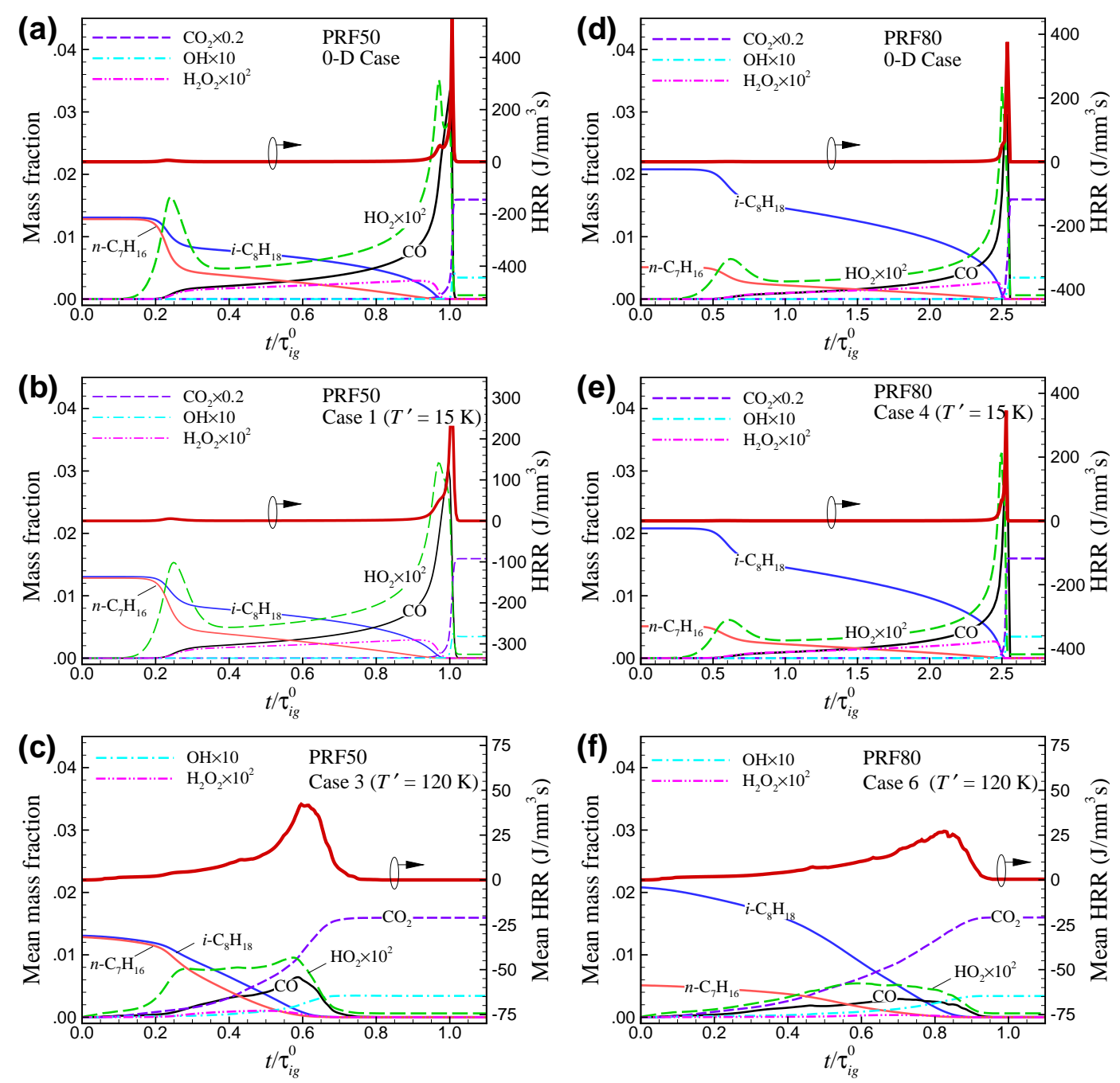

Figure 4: Temporal evolutions of the mean mass fractions of important species and mean HRR for (a) 0-D case of PRF50/air mixture, (b) Case 1, (c) Case 3, (d) 0-D case of PRF80/air mixture, (e) Case 4, and (f) Case 6.
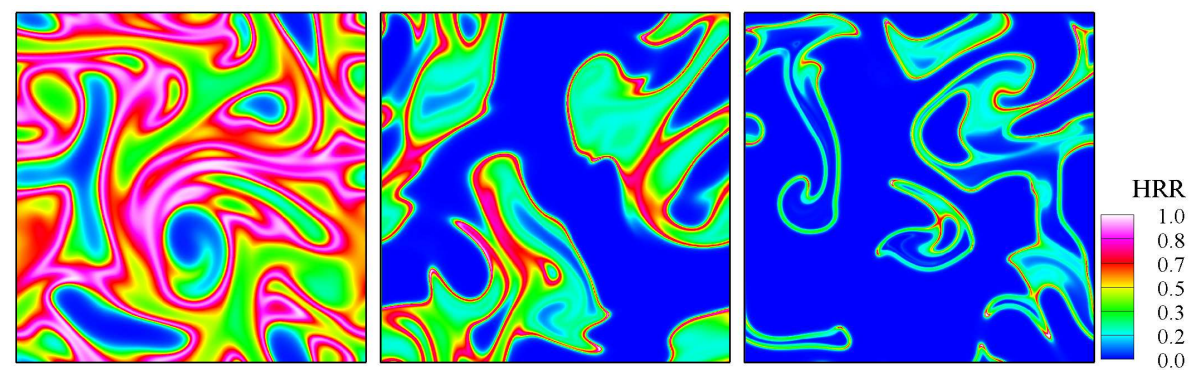

Figure 5: Isocontours of the normalized heat release rate for Cases $1-3$ (from left to right) at $t / \tau_{i g}^{0}=1.0,0.94$, and 0.61 , respectively. 
(a) $\operatorname{sign}\left(\lambda_{\text {exp }}\right) \times \log _{10}\left(\max \left(1, \lambda_{\text {exp }}\right)\right.$

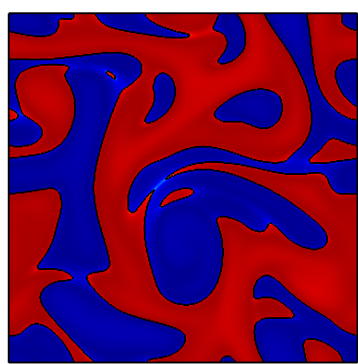

\begin{tabular}{llllllll}
\hline & & & & & & & \\
-6.0 & -4.0 & -2.0 & 0.0 & 2.0 & 4.0 & 6.0
\end{tabular}

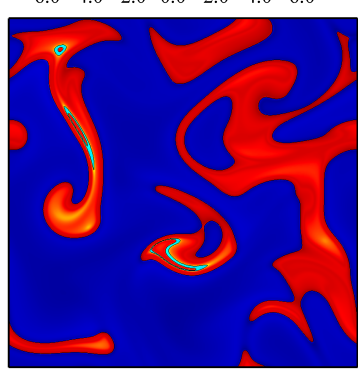

(b) $\operatorname{sign}\left(\lambda_{\text {exp }}\right) \times \log _{10}(\max (1,|\mathrm{Da}|))$
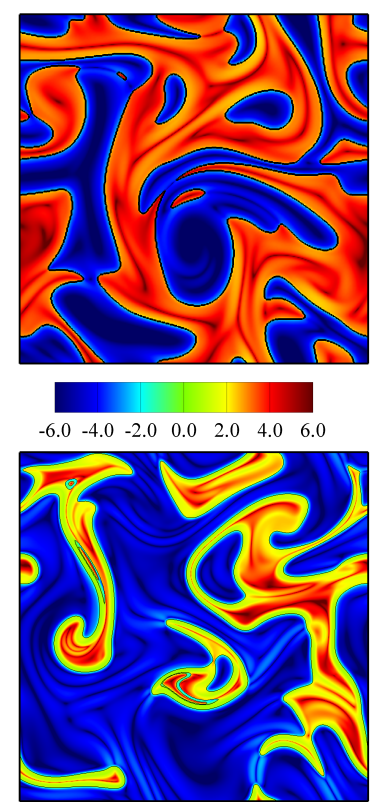

(c) Temperature $(1000 \mathrm{~K})$
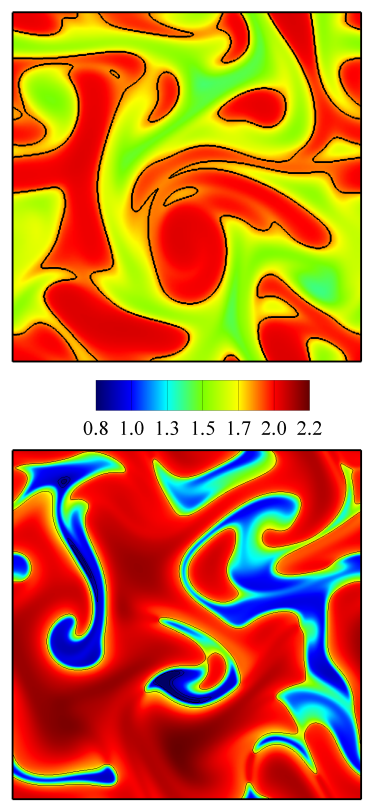

(d) $\log _{10}(1+\chi)$

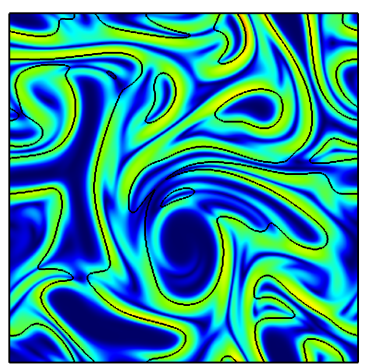

\begin{tabular}{lllllll}
\hline 0.0 & 0.7 & 1.3 & 2.0 & 2.7 & 3.3 & 4.0
\end{tabular}

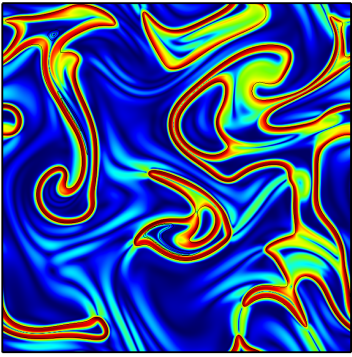

Figure 6: Isocontours of (a) the timescale of the chemical explosive mode, (b) $D a_{c}$, (c) temperature, and (d) $\chi$ for Case 1 (top) at $t / \tau_{i g}^{0}=1.0$ and Case 3 (bottom) at $t / \tau_{i g}^{0}=0.61$. The solid line denotes the reaction front $\left(D a_{c}=\right.$ $1)$.
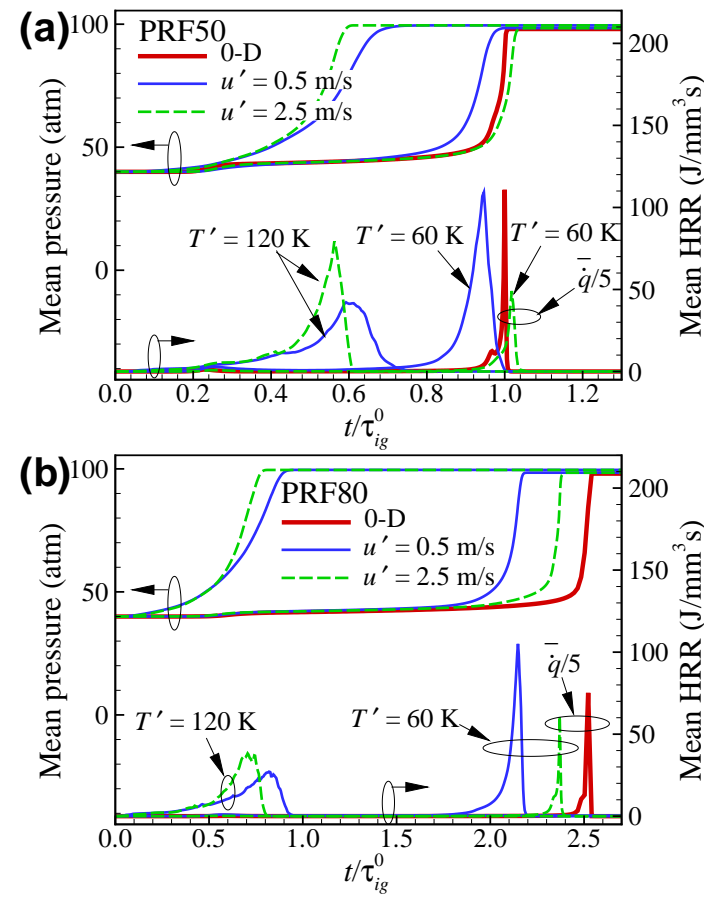

Figure 7: Temporal evolutions of the mean pressure and mean HRR for (a) PRF50 and (b) PRF80. 

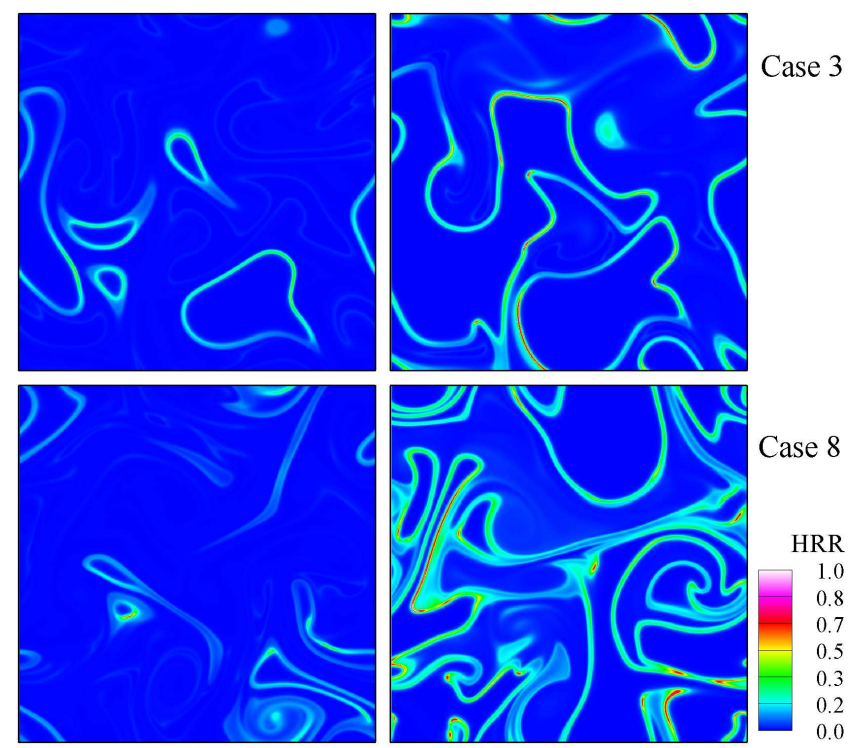

Figure 8: Isocontours of the normalized heat release rate for Cases 3 (top) and 8 (bottom). From left to right, $t / \tau_{i g}^{0}$ $=0.36$ and 0.52 , respectively.
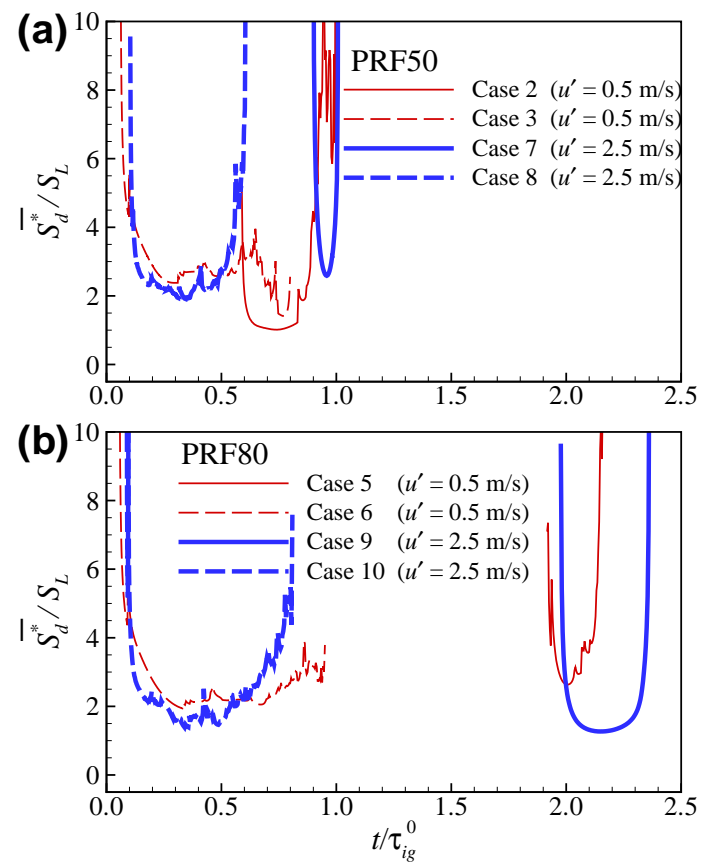

Figure 9: Temporal evolution of the mean front speed, $\overline{S_{d}^{*}}$, for (a) Cases 2, 3, 7, and 8 (PRF50) and (b) Cases 5, 6, 9, and 10 (PRF80). 


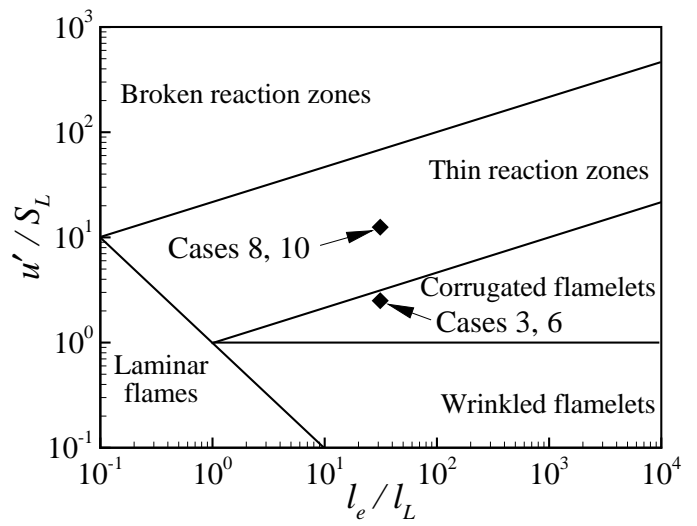

Figure 10: The locations of the cases with small $u^{\prime}$ (Cases 3 and 6) and large $u^{\prime}$ (Cases 8 and 10) in the regime diagram for turbulent premixed combustion. 